\title{
Abstracts
}

\section{Transition/Adult Care Session 2}

\author{
Perceptions of Healthcare by Adults with Spina \\ Bifida \\ Still, Monica A. \\ SBAA
}

Background Since the early 1960's infants born with Spina Bifida have grown into adults. In 2006 the CDC began preliminary studies about healthcare services for adults with SB. Focus has been on the healthcare community's perception of the care available for adults with SB. This researcher could not find any study that addressed how the adult with SB perceives available healthcare.

Methods During the 2016 SBAA National Conference this researcher documented the anecdotal accounts of healthcare experiences of 150 adults with SB.

Results Common themes:

Encountered ER staff who had no knowledge of adults with SB

Encountered clinicians who assumed no one lived past 20

Encountered clinicians who assumed all adults with SB were wc users

Encountered clinicians who assumed the adult with SB was the patient not the caregiver

Reported women's health issues being ignored

Reported being told they could not have children because of SB

Reported difficulty finding an adult neurologist versed in $\mathrm{SB}$

Reported continuing to see a pediatrician for neurology after the age of 21

Felt clinicians did not understand they wished to maintain function

Felt that they were educating the medical community about SB

Voiced concern that their care was not meeting their needs

Think they are aging faster than their able bodied counterparts
Reported physical accessibility and transportation issues impeding access to healthcare

Felt the medical community did not realize that they had a life beyond being a patient

Had met doctors that did not realize that SB was present since birth

Felt the medical community lacked knowledge of aging with $\mathrm{SB}$

Conclusions The medical community needs to improve education of clinicians about SB beyond young adulthood. Studies need to focus on aging and SB. Adults with SB need tools to independently manage their healthcare. Adults with SB need to be given a voice in determining their own healthcare needs. Barriers to access to healthcare need to be identified and addressed.

\section{Spina Bifida Transition to Adulthood \\ Hopson, Betsy D. ${ }^{1}$, Rocque, Brandon G. ${ }^{1}$, Wilson, Tracey S. ${ }^{2}$, Powell, Danielle ${ }^{2}$, McLain, Amie B. ${ }^{2}$, Joseph, David B. ${ }^{1}$, Blount, Jeffrey ${ }^{1}$ \\ ${ }^{1} U A B$-Children's of Alabama \\ ${ }^{2} U A B$}

Background In October of 2010 Children's of Alabama (COA) partnered with the University of Alabama at Birmingham to develop a transition/adult Spina Bifida clinic. During the initial phase of development, the clinic was modeled after the existing pediatric multi-disciplinary clinic but we quickly determined that there were areas of deficiency in the adult clinic and opportunities to improve the outcomes of adult patients.

Methods After our anecdotal observation that the transitioning population was growing in depression and loss of motivation, we designed multiple studies to determine key areas of importance to adults with spina bifida. In addition, we began to systematically study transition readiness. 
Results We found that there are 4 areas that have great impact on young adults in our clinic; lack of employment (57\% indentify as permanently disabled), bowel management (49\% have accidents monthly or greater), obesity $(55 \%>30 \mathrm{BMI})$, and skin breakdown $(30 \%$ reported skin breakdown either active or within the previous 12 months of their visit). We also learned that only $38 \%$ of the patients seen in our transition/adult clinic were transitioned from COA. Thus, the majority of patients seen in the adult clinic likely did not have a multidisciplinary, spina bifida-focused approach to their health care as adults prior to their arrival at our clinic. Finally, our readiness assessment scores showed that to improve transition readiness, transition teaching needed to begin earlier than age 19 .

Conclusions Based on these results, we have reduced the age of transition teaching from age 19 to age 14. We have learned the importance of a two-way education exchange between the pediatric and adult care providers. The pediatric team educates the adult providers, and the adult providers give information to the pediatric team on ways independence can be improved where transition education efforts should focus. Future studies are needed in depression/anxiety and sexuality in this population.

\section{Self-Management in Spina Bifida: A Synthesis of the Literature}

Sawin, Kathleen J. ${ }^{1,2}$, Bellin, Melissa H. ${ }^{3}$, Woodward, Jason $^{4}$, Brei, Timothy ${ }^{5,6}$

${ }^{1}$ Children's Hospital of Wiscosin

${ }^{2}$ University of Wisconsin-Milwaukee

${ }^{3}$ University of Maryland

${ }^{4}$ Cincinnati Children's Hospital Medical Center

${ }^{5}$ Seattle Children's Hosptial

${ }^{6}$ University of Washington

Background Developing the skills for Spina Bifida (SB) self-management is a central task for adolescents and young adults transitioning into adulthood. Three self-management research priorities were delineated in the 2003 SB Research Agenda; (1) Assessment of ways to measure self-management (2) Factors that affect the teaching and learning of self-management and (3) Optimizing use of assistive devices. The purpose of this study was to identify studies that address these priorities published from 2003-2016.

Methods A literature search was conducted using PRISMA guidelines. The Medline, CIHNL and PsychINFO data bases with searched using the terms spina bifida (or myelomeningocele) and self-management. Articles were included if they addressed self-management, or self-care and were written in English. Studies were omitted if they used a combined disability sample where the sample of individuals with SB was not specifically reported.

Results Of the twenty-three manuscripts identified, three were intervention-based with varying degrees of success in advancing self-management behaviors. A workshop intervention failed to impact self-management behaviors but a mobile health intervention did improve self-management, especially for those who were high users of the technology. Four measures of self-management have been developed: (1) Adolescent Self-Management and Independence Scale II, (2) Kennedy Krieger Independence Scales (SB), (3) SB Self-Management Profile, (4) Sharing of SB Management Responsibilities Scale, each with a specific focus and preliminary psychometrics. Factors associated with self-management included age, severity of SB, self-efficacy, chores, decision making, and SB knowledge.

Conclusions Progress in advancing self-management research priorities is noted in instrument development but additional research to test self-management interventions is indicated.

Pain and health status in adults with myelomeningocele living in southern Sweden

Alriksson-Schmidt, Ann ${ }^{1,3}$, Josenby-Lundkvist Annika $^{2}$, Lindquist, Barbro ${ }^{4}$, Westbom, Lena ${ }^{5}$

${ }^{1}$ Skåne University Hospital

${ }^{2}$ Lund University Department of Health Sciences

${ }^{3}$ Lund University, Dept. of Clinical Sciences, Orthopedics

${ }^{4}$ The Habilitation Center, Hospital of Halland

${ }^{5}$ Lund University, Department of Clinical Sciences

Lund, Paediatrics

Background Little attention has been paid to pain in myelomeningocele (MMC). The few studies that exist have reported contradictory findings. Individuals with MMC frequently have risk factors for pain such as musculoskeletal deformities, clogged/infected shunts, urinary tract infections, bowel problems, and suboptimal positioning. Individuals with $\mathrm{MMC}$ often undergo surgeries, which may also result in pain. We investigated pain and health status in adults with MMC.

Methods Current health status was measured using a visual analogue scale (VAS, 0-100) from EQ5D- 
$5 \mathrm{~L}$, ranging from 0 to 100 . The presence and location of pain, and how the pain interfered with sleep/work were measured. Fifty-one participants were included $(53 \%=$ males, age; median age $=29$ years $)$. Frequencies (n \%) and descriptive statistics (median, SD) were calculated. A GLM univariate ANOVA was used to analyze the association between gender, age and current health status.

Results Thirty-one participants reported pain in the past 4 weeks. There was no significant difference on pain based on sex. Pain was reported in the abdomen (3), shoulders (14), arms/hands (4), hips (7), knee/femur (7), lower leg/feet (4), and head (7). Eight participants reported that pain interfered with sleep "moderately" to "a lot", and 12 that pain interfered with their daily work "moderately" to "very much". The average score on health status was 70.90 (SD = 20.91). The ANOVA revealed significant main effects for both sex and age on the health score, $\mathrm{F}$ $(2,44)=5.63, p=0.007$, adjusted $\mathrm{R}^{2}=0.17$. Women had a lower average score on current health (adjusted average $=63.58$ ) than men (adjusted average $=76.33$ ). Older persons reported significantly lower current health scores than did younger $(\mathrm{B}=-89$, $\mathrm{t}=-2.79, p=0.008)$.

Conclusions Pain was frequently reported and the location of pain differed widely. Women scored significantly lower on health status as did older persons.

Effectiveness of a Wellness Program for Individuals with Spina Bifida and Spinal Cord Injuries within an Integrated Delivery System

Dicianno, Brad E. ${ }^{1}$, Lovelace, John ${ }^{2}$, Peele, Pamela ${ }^{2,3}$, Fassinger, Christopher ${ }^{2}$, Houck, Patricia ${ }^{2}$, Bursic,

Alexandra $^{1}$, Boninger, Michael ${ }^{1}$

${ }^{1}$ University of Pittsburgh School of Medicine

${ }^{2}$ UPMC Center for High-Value Health Care

${ }^{3}$ University of Pittsburgh

Background Individuals with spina bifida (SB) and spinal cord injury (SCI) experience a high rate of potentially preventable conditions such as urinary tract infections, pressure ulcers and sepsis. These secondary complications are responsible for up to $47 \%$ of hospitalizations and over a third of emergency department visits. This study's objective was to determine whether an evidence-based wellness program would improve health outcomes and patient experience of care, result in a positive return on investment (ROI), and provide evidence for scalability.
Methods Individuals with SCI and SB enrolled in a non-randomized, non-controlled cohort study for two years at an academic hospital-based outpatient physiatry clinic partnered with an insurance division within an integrated healthcare delivery and financing system. They participated in an evidence-based wellness program consisting of care coordination from a mobile nurse, patient education, and patient incentives. Outcome measures were validated measures of function, mood, quality of life, and perception of care delivery; knowledge of preventable conditions; self-rating of health; utilization and cost.

Results Sixty nine individuals with SB and SCI were consented, 4 were excluded, and the remaining 65 participated in the intervention. Improvements in all main outcome measures were seen after two years of enrollment. Although cost in year 1 of enrollment increased due to hospitalizations, and the overall ROI was negative, a small positive ROI was seen in year 2 of enrollment.

Conclusions Participation in an evidence-based wellness program was associated with improved health and experience of care. Scaling the program to larger numbers may result in an overall positive ROI.

This study was funded by the UPMC Health Plan and the UPMC Rehabilitation Institute Pilot Funds Program.

\section{Spina Bifida in Iceland: Epidemiology, Health and} Well-being among adults

Meintema, Marrit ${ }^{1}$, Sigurdardóttir, Solveig ${ }^{1}$, Árnadóttir, Solveig Á. ${ }^{2}$, Gudjónsdóttir, Björg ${ }^{2}$

${ }^{1}$ State Diagnostic and Counselling Centre

${ }^{2}$ Department Of Physical Therapy, University Of Iceland

Background Spina bifida (SB) is a congenital deformity with complications that require comprehensive multidisciplinary care. Physical limitations and complications associated with SB might set individuals with SB at increased risk for developing lifestylerelated conditions

Aim: To perform an epidemiological investigation of the SB population in Iceland and to assess health and well-being of the adult SB population.

Methods The incidence of SB between 1972 and 2011 was examined retrospectively. Mobility and cognitive functioning of children with SB at 5 years of age was described. In addition, 25 adults with SB answered the survey "Health and well-being of Icelanders in 2012" 
and the results were compared with results from a group of Icelandic participants $(n=2159)$. They also wore an accelerometer for 7 days to measure physical activity (PA). Waist circumference was measured to evaluate risk of metabolic complication.

Results The incidence of SB has declined significantly in Iceland during the last 40 years. Most 5 year old children with SB could walk. The results concerning health and well-being of adults with SB showed that most of them considered their health as being good or very good and similar or better than last year. They drank less alcohol than comparison group but tended to eat unhealthily. They did not carry out moderately intense PA for 30 minutes a day. Most of them had an increased waist circumference.

Conclusions Induced abortion is the main reason for decline in incidence. Physiotherapists (PTs) can play an important role in improving and prolonging gait function of children with SB. Adults with SB are at increased risk of developing life-style related conditions because of poor diet, lack of PA and prolonged sitting. PTs should inform and educate clients with SB and their families about the importance of a healthy diet and promote PA which takes into account individual possibilities, preferences and limitations.

Feasibility of using Mobile Health to Promote Selfmanagement in Spina Bifida

Dicianno, Brad E. ${ }^{1}$, Fairman, Andrea ${ }^{2}$, McCue,

Michael $^{1}$, Parmanto, Bambang ${ }^{1}$, Yih, Erika ${ }^{1}$, McCoy,

Andrew $^{1}$, Pramana, Gede ${ }^{1}$, Yu, Daihua ${ }^{1}$, McClelland, Justin $^{1}$, Collins, Diane ${ }^{3}$, Brienza, David ${ }^{1}$

${ }^{1}$ University of Pittsburgh

${ }^{2}$ MGH Institute of Health Professions

${ }^{3}$ University of Texas Medical Branch
Background The objective of this study was to determine the feasibility of using the interactive Mobile Health and Rehabilitation (iMHere) system in spina bifida and its effects on psychosocial and medical outcomes.

Methods In a randomized controlled trial, 13 intervention participants using the iMHere system and receiving usual care and 10 control participants receiving usual care were followed for one year.

Results Feasibility of use of the system was demonstrated by participants using a customized smartphone system for reminders to conduct various self-care tasks, upload photos of wounds, manage medications, complete mood surveys, and for secure messaging. High utilization of the system was associated with positive changes in the subscales of the Adolescent SelfManagement and Independence Scale II.

Conclusions Use of the iMHere system in spina bifida is feasible and was associated with short-term selfreported improvements in self- management skill. This system holds promise for use in many diverse chronic care models to support and increase self-management skills.

This study was funded by the National Institute on Disability and Rehabilitation Research (Rehabilitation Engineering Research Centers on Telerehabilitation H133E090001), the Verizon Foundation, National Institute on Disability, Independent Living, and Rehabilitation Research (NIDILRR) grant \#H133E140039 (RERC From Cloud to Smartphone: Empowering and Accessible ICT) and grant \#H133A140005 (DRRP Promoting Independence \& Self-management using mHealth), National Institutes of Health (NIH) grant \#1R21HD071810-01A1, and National Institutes of Health 5T35AT005933-02. 